Article

\title{
Association of the MicroRNA-146a SNP rs2910164 with Ischemic Stroke Incidence and Prognosis in a Chinese Population
}

\author{
Jiao-Yan Qu ${ }^{1,2,+}$, Jie Xi ${ }^{1,+}$, Yin-Hui Zhang ${ }^{1}$, Chan-Na Zhang ${ }^{1}$, Li Song ${ }^{1}$, Yan Song ${ }^{1}$, \\ Ru-Tai Hui ${ }^{1}$ and Jing-Zhou Chen ${ }^{1, *}$ \\ 1 Sino-German Laboratory for Molecular Medicine, State Key Laboratory of Cardiovascular Disease, \\ National Center for Cardiovascular Diseases, Fuwai Hospital, Chinese Academy of Medical Sciences, \\ 167 Beilishilu, Beijing 100037, China; qujiaoyan1989@163.com (J.-Y.Q.); xijie226688@163.com (J.X.); \\ zyh0863@163.com (Y.-H.Z.); china800908@hotmail.com (C.-N.Z.); lisong97@aliyun.com (L.S.); \\ yansong1234@hotmail.com (Y.S.); huirutai@sglab.org (R.-T.H.) \\ 2 Department of Hematology, Beijing Luhe Hospital Affiliated to Capital Medical University, \\ Beijing 101149, China \\ * Correspondence: chendragon1976@aliyun.com or chenjingzhou@fuwaihospital.org; Tel.: +86-10-6086-6383; \\ Fax: +86-10-6086-6091 \\ + These authors contributed equally to this study.
}

Academic Editor: William Chi-shing Cho

Received: 9 March 2016; Accepted: 23 April 2016; Published: 5 May 2016

\begin{abstract}
We conducted a case-control study investigating the association between the singlenucleotide polymorphism rs2910164 in microRNA (miR)-146a and the risk and prognosis of stroke. We recruited a total of 1139 ischemic stroke patients and 1585 sex- and age-matched control subjects. After a median follow-up period of 4.5 years, 1071 of these ischemic stroke patients were then recruited for a prospective study. Our study revealed that rs2910164 was not associated with ischemic stroke incidence (odds ratio $=1.00 ; 95 \%$ confidence interval $(\mathrm{CI})=0.80-1.24 ; p=0.985$ ) by multivariate logistic regression. Meta-analysis of our case-control study and three others on Asian populations also suggested that there was no relationship between rs2910164 and ischemic stroke incidence. The significance of differences in long-term outcomes was examined by the log-rank test of the respective comparison groups. The prospective study showed that rs2910164 led to a 1.56-fold increased risk of stroke recurrence (hazard ratio $(\mathrm{HR})=1.56 ; 95 \% \mathrm{CI}=1.10-2.20 ; p=0.013$ ) and a 2.13-fold increased risk of death caused by cardiovascular disease or stroke (Csdeath) ( $\mathrm{HR}=2.13$; $95 \% \mathrm{CI}=1.31-3.46 ; p=0.002$ ). The independent association of $\mathrm{rs} 2910164$ with stroke prognosis was evaluated using Cox regression models. Therefore, rs2910164 appears to be a strong predictor of stroke prognosis but not of stroke incidence in Asian populations.
\end{abstract}

Keywords: incidence; miR-146a; polymorphisms; prognosis; stroke

\section{Introduction}

Stroke has a limited therapeutic time window and a very high rate of recurrence, and thus is a leading cause of death and constitutes a heavy economic burden in many countries, including China [1-4]. It is a multifactorial disease affected by environmental and genetic risk factors including hypertension, diabetes mellitus, smoking, hyperlipidemia, and hyperhomocysteinemia [5,6]. Multiple susceptibility genes have been demonstrated to have a relationship with an enhanced risk of stroke or worse stroke prognosis, including $F 5$, angiotensin-converting enzyme ( $A C E)$, methylenetetrahydrofolate reductase (MTHFR), serpin peptidase inhibitor 1 (SERPINE1), apolipoprotein E (APOE) [7], cytochrome P450 2C19 (CYP2C19) [8], and platelet-derived growth factor D (PDGF-D) [9], as well as chromosome 12p13 
variants [10]. However, the genetic factors identified cannot fully explain the observed inherited risk of stroke.

MicroRNAs (miRNAs) are a class of endogenous, small, 22-nucleotide non-coding RNAs attached to the $3^{\prime}$-untranslated regions of mRNAs through highly conserved seed sequences, and are known to negatively regulate mRNA expression. Genetic alterations in miR sequences impact on precursor processivity, maturation, expression, and ultimately influence the expression of target mRNAs [11-13]. miRNAs are important in biological processes including cell differentiation, proliferation, growth, stress resistance, and metabolism, as well as the pathophysiology of neurodegenerative disease, cancer, and cardiovascular disease [14-16]. Emerging evidence also indicates that circulating miRNAs may be novel biomarkers for the diagnosis and prognosis of stroke $[17,18]$. This reflects their role in modulating transcriptional programs that affect the processes of atherosclerosis, including endothelial integrity, inflammation, and extracellular matrix remodeling $[19,20]$.

An important and universal type of genetic variation are single-nucleotide polymorphisms (SNPs) [21], which may affect miRNA function by modulating biogenesis or target selection [22]. Recently, a well-documented common polymorphism in a pre-miRNA sequence (miR-146a C > G (rs2910164; chromosome 5, 160485411)) was found to be involved in a variety of diseases [23-26]. This variant altered the specific base pairing of the stem region, which influenced the expression of mature miR-146a [27]. This binds to target mRNAs including tumor necrosis factor- $\alpha$ (TNF- $\alpha$ ) [28], C-reactive protein and interleukin-1 receptor-associated kinase-1 (IRAK1) [29], which affect vascular damage responses and inflammation-related atherosclerosis in the development of stroke. However, studies into the relationship between the miR-146a rs2910164 SNP and ischemic stroke in different ethnic populations have provided conflicting results.

To investigate this in greater detail, we designed a case-control study of ischemic stroke patients in a Chinese population and a meta-analysis of four case-control studies into the role of rs2910164 in Asian ischemic stroke patients. A prospective study was also conducted to evaluate the effects of rs2910164 on stroke prognosis.

\section{Results}

\subsection{Baseline Characteristics}

The baseline characteristics of the ischemic stroke patients and control groups are listed in Table 1. After a follow-up (median time, 4.5 years) of 1071 ischemic stroke patients, a total of 196 recurrent strokes were recorded. The CC, GC, and GG rs2910164 genotype frequencies were $31.2 \%, 54.3 \%$, and $14.5 \%$ for patients, and $30.5 \%, 54.8 \%$, and $14.7 \%$ for controls, respectively (Table 2 ). SNP genotype distributions in both groups followed the Hardy-Weinberg equilibrium (HWE).

Table 1. Baseline characteristics of the study population.

\begin{tabular}{cccc}
\hline Characteristics & Control $(\boldsymbol{n}=\mathbf{1 5 8 5})$ & Ischemic Stroke $(\boldsymbol{n}=\mathbf{1 1 3 9})$ & $\boldsymbol{p}$-Value \\
\hline Age (years) & $59.50 \pm 8.50$ & $61.30 \pm 9.40$ & $<0.001$ \\
Male $(\%)$ & $903(57)$ & $718(63)$ & 0.004 \\
SBP, $\mathrm{mmHg}$ & $128.86 \pm 17.68$ & $145.43 \pm 22.26$ & $<0.001$ \\
$\mathrm{DBP}, \mathrm{mmHg}$ & $79.55 \pm 9.79$ & $86.24 \pm 12.36$ & $<0.001$ \\
TC, $\mathrm{mmol} / \mathrm{L}$ & $5.17 \pm 1.29$ & $5.07 \pm 1.20$ & 0.620 \\
TG, $\mathrm{mmol} / \mathrm{L}$ & $1.56 \pm 1.15$ & $1.90 \pm 1.56$ & $<0.001$ \\
HDL-C, mmol/L & $1.44 \pm 0.42$ & $1.23 \pm 0.44$ & $<0.001$ \\
Glucose, mmol/L & $5.69 \pm 1.83$ & $6.28 \pm 2.96$ & $<0.001$ \\
\hline Cigarette smoking $(\%)$ & & & $<0.001$ \\
\hline Never & $992(62.60)$ & $584(51.30)$ & $<0.001$ \\
Former & $201(12.70)$ & $251(22.00)$ & $<0.001$ \\
Current & $392(24.70)$ & $304(26.70)$ &
\end{tabular}


Table 1. Cont.

\begin{tabular}{cccc}
\hline Characteristics & Control $(\boldsymbol{n = 1 5 8 5 )}$ & Ischemic Stroke $(\boldsymbol{n = 1 1 3 9 )})$ & $p$-Value \\
\hline Alcohol intake $(\%)$ & & & \\
\hline Nondrinker & $1453(91.7)$ & $936(82.2)$ & $<0.001$ \\
Drinker & $132(8.3)$ & $203(17.8)$ & $<0.001$ \\
Hypertension history (\%) & $412(26.00)$ & $714(62.70)$ & $<0.001$ \\
DM history (\%) & $86(5.40)$ & $177(15.50)$ & $<0.001$ \\
\hline
\end{tabular}

SBP: Systolic blood pressure; DBP: Diastolic blood pressure; TC: Total plasma cholesterol; TG: Triglyceride; HDL-C: High-density-lipoprotein cholesterol; DM: Diabetes mellitus.

Table 2. Genotype and allele frequencies of rs2910164.

\begin{tabular}{cccc}
\hline Genotype & No. of Controls (\%) & No. of Strokes (\%) & $p$-Value \\
\hline C/C & $483(30.5)$ & $355(31.2)$ & \\
G/C & $869(54.8)$ & $618(54.3)$ & \\
G/G & $233(14.7)$ & $166(14.5)$ & \\
Total & $1585(100)$ & $1139(100)$ & 0.928 \\
\hline
\end{tabular}

\section{2. rs2910164 Is Not Associated with Ischemic Stroke Incidence}

Under the dominant model, the GG + CG genotype of rs2910164 was not associated with ischemic stroke incidence compared with the CC genotype (OR $=1.00,95 \% \mathrm{CI}=0.78-1.27, p=0.961)$ after adjustment for Model 3. For the cerebral thrombosis subgroup, no association between rs2910164 and ischemic stroke incidence ( $\mathrm{OR}=1.02,95 \% \mathrm{CI}=0.84-1.24, p=0.834)$ after adjustment for Model 3, as was the case for the lacunar infarct subgroup (OR $=0.96,95 \% \mathrm{CI}=0.76-1.22, p=0.745)$ (Table 3). The rs2910164 genotype was not connected with the ischemic stroke incidence according to sex after adjustment for Model 2 (Table 4).

Under the recessive model, the rs2910164 GG genotype was not associated with ischemic stroke incidence compared with the GC + CC genotype (OR $=1.00,95 \% \mathrm{CI}=0.80-1.24, p=0.985)$ after adjustment for Model 3. For the cerebral thrombosis subgroup, no association between rs2910164 and ischemic stroke incidence ( $\mathrm{OR}=0.99,95 \% \mathrm{CI}=0.77-1.28, p=0.950)$ after adjustment for Model 3 , as was the case for the lacunar infarct subgroup ( $\mathrm{OR}=1.02,95 \% \mathrm{CI}=0.75-1.38, p=0.922)$ (Table 3 ). The rs2910164 genotype was not associated with the ischemic stroke incidence according to sex after adjustment for Model 2 (Table 4).

\subsection{Meta-Analysis of the Relationship between rs2910164 and Ischemic Stroke Incidence}

A forest plot was constructed from the findings of four studies showing the relationship between rs2910164 and ischemic stroke in Asian populations under the dominant model (genotype (GG + CG) vs. CC) (Figure 1). This suggested that rs2910164 does not affect the ischemic stroke incidence $(\mathrm{OR}=1.01,95 \% \mathrm{CI}=0.90-1.14)$.

\section{4. rs2910164 Is a Strong Predictor of Stroke Prognosis}

This prospective study showed that the rs2910164 GG genotype notably increased the risk of stroke recurrence (Figure 2a, $p=0.016$ ). Kaplan-Meier estimates of the cumulative recurrence-free probability in ischemic stroke patients based on the $C$ allele of rs2910164 polymorphism were: log-rank statistic $\chi^{2}=5.796 ; p=0.016$. Cox proportional hazards analysis indicated that the GG genotype had a 1.56-fold increased risk for recurrence (Table 5, HR $=1.56,95 \% \mathrm{CI}=1.10-2.20, p=0.013$ ) after adjusting for Model 3. Additionally, the GG genotype significantly increased the risk of death caused by cardiovascular disease or stroke (Csdeath) (Figure 2b, $p=0.002$ ). Kaplan-Meier estimates of the cumulative event-free survival probability in ischemic stroke patients based on the $C$ allele of rs2910164 polymorphism were: log-rank statistic $\chi^{2}=9.155, p=0.002$. The analysis of Cox proportional hazards showed that the GG genotype had a 2.13-fold increased risk for Csdeath (Table 5, HR = 2.13, $95 \% \mathrm{CI}=1.31-3.46, p=0.002$ ) after adjusting for Model 3 . 
Table 3. rs2910164 genotype was not associated with ischemic stroke incidence.

\begin{tabular}{|c|c|c|c|c|c|c|c|c|}
\hline \multirow{2}{*}{ Variant Group (Number) } & \multicolumn{2}{|c|}{ Genotype Number (\%) } & \multicolumn{2}{|c|}{ Model 1} & \multicolumn{2}{|c|}{ Model 2} & \multicolumn{2}{|c|}{ Model 3} \\
\hline & & & OR $(95 \% \mathrm{CI})$ & $p$-Values & OR $(95 \% \mathrm{CI})$ & $p$-Values & OR $(95 \% \mathrm{CI})$ & $p$-Values \\
\hline Dominant model & $\mathrm{CC}$ & $\mathrm{GG}+\mathrm{CG}$ & & & & & & \\
\hline Control (1585) & $483(30.5)$ & $1102(69.5)$ & 1.00 & & 1.00 & & 1.00 & \\
\hline Stroke (1139) & $355(31.2)$ & $784(68.8)$ & $1.03(0.81-1.31)$ & 0.801 & $0.98(0.77-1.25)$ & 0.870 & $1.00(0.78-1.27)$ & 0.961 \\
\hline Cerebral thrombosis (718) & $223(31.1)$ & $495(68.9)$ & $0.97(0.80-1.18)$ & 0.778 & $1.03(0.84-1.25)$ & 0.792 & $1.02(0.84-1.24)$ & 0.834 \\
\hline Lacunar infarct (421) & $132(31.4)$ & $289(68.6)$ & $0.96(0.76-1.21)$ & 0.728 & $0.96(0.76-1.21)$ & 0.707 & $0.96(0.76-1.22)$ & 0.745 \\
\hline Recessive model & $\mathrm{GC}+\mathrm{CC}$ & GG & & & & & & \\
\hline Control (1585) & $1352(85.3)$ & $233(14.7)$ & 1.00 & & 1.00 & & 1.00 & \\
\hline Stroke (1139) & $973(85.4)$ & $166(14.6)$ & $0.99(0.80-1.23)$ & 0.927 & $1.02(0.82-1.27)$ & 0.868 & $1.00(0.80-1.24)$ & 0.985 \\
\hline Cerebral thrombosis (718) & $613(85.4)$ & $105(14.6)$ & $1.01(0.78-1.29)$ & 0.962 & $0.97(0.75-1.25)$ & 0.813 & $0.99(0.77-1.28)$ & 0.950 \\
\hline Lacunar infarct (421) & $360(85.5)$ & $61(14.5)$ & $0.98(0.73-1.33)$ & 0.913 & $0.99(0.73-1.35)$ & 0.971 & $1.02(0.75-1.38)$ & 0.922 \\
\hline
\end{tabular}

Abbreviations: OR: Odds ratio; CI: Confidence interval. Model 1: Unadjusted OR; Model 2: Adjusted for age and sex; Model 3: Adjusted for age, sex, hypertension, diabetes mellitus, smoking status, and alcohol intake (adjusted ORs (95\% CI) and adjusted $p$-values were computed using multivariate logistic regression analyses).

Table 4. rs2910164 genotype was not associated with ischemic stroke incidence according to sex.

\begin{tabular}{ccccc}
\hline \multirow{2}{*}{ Variant Group } & \multicolumn{2}{c}{ Model 1 } & \multicolumn{2}{c}{ Model 2 } \\
\cline { 2 - 5 } & OR (95\% CI) & $p$-Value & OR (95\% CI) & $p$-Value \\
\hline Dominant model & & & & \\
\hline Sex & & & & \\
Male & $0.81(0.65-1.00)$ & 0.049 & $0.84(0.68-1.04)$ & 0.109 \\
Female & $1.25(0.96-1.63)$ & 0.097 & $1.31(1.00-1.73)$ & 0.052 \\
\hline Recessive model & & & & \\
\hline Sex & & & & \\
Male & $0.97(0.74-1.28)$ & 0.840 & $0.97(0.73-1.28)$ & 0.821 \\
Female & $1.07(0.76-1.50)$ & 0.709 & $1.05(0.74-1.50)$ & 0.771 \\
\hline
\end{tabular}

Abbreviations: OR: Odds ratio; CI: Confidence interval. Model 1: Unadjusted OR; Model 2: Adjusted for age, hypertension, diabetes mellitus, smoking status, and alcohol intake (adjusted ORs $(95 \% \mathrm{CI})$ and adjusted $p$ values were computed using multivariate logistic regression analyses). 


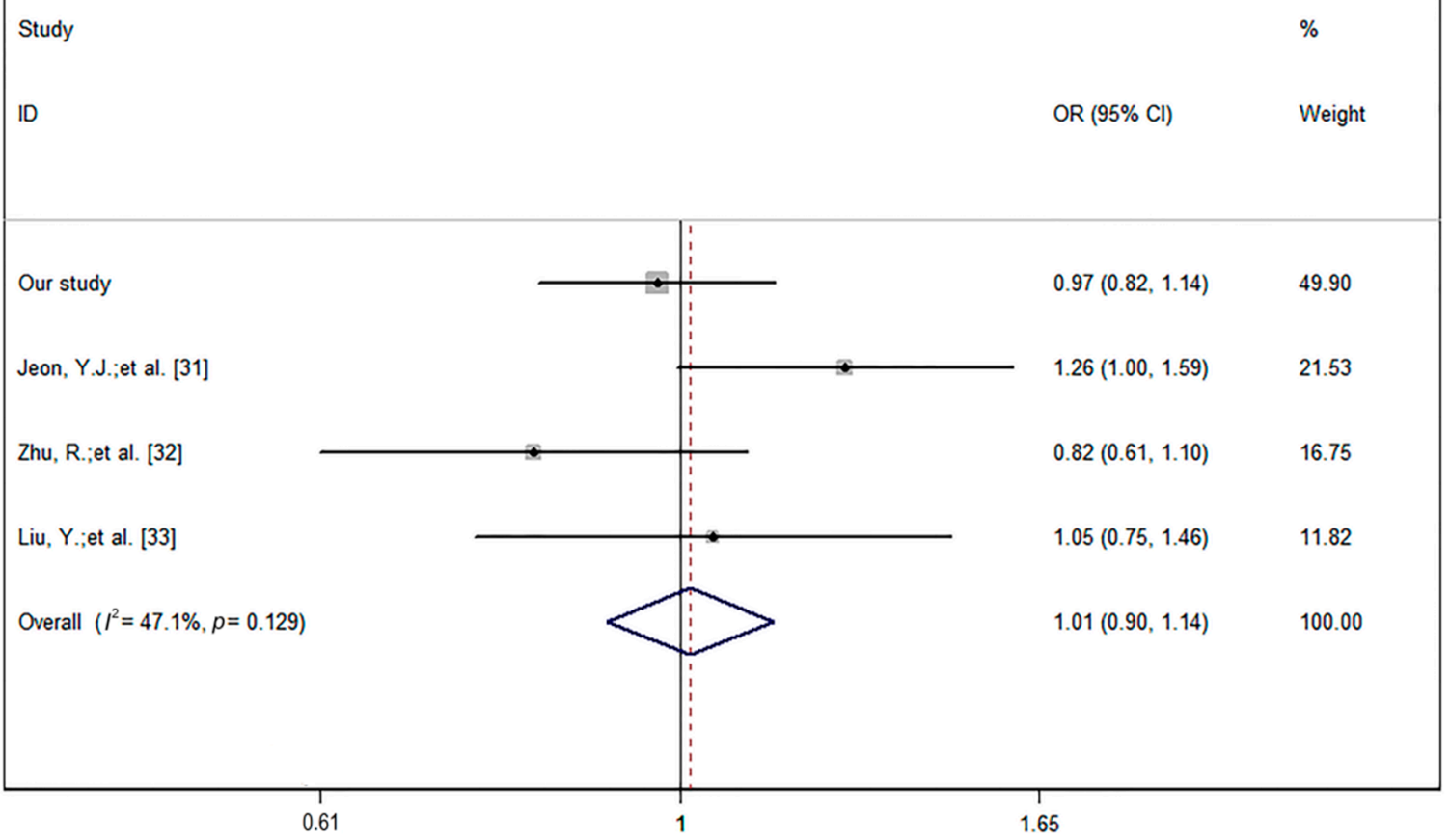

Figure 1. Forest plot showed the relationship between rs2910164 and ischemic stroke in Asian populations (four studies) under the dominant model (genotype (GG + CG) vs. CC). Heterogeneity: $\chi^{2}=5.67, p=0.129, I^{2}=47.1 \%$.
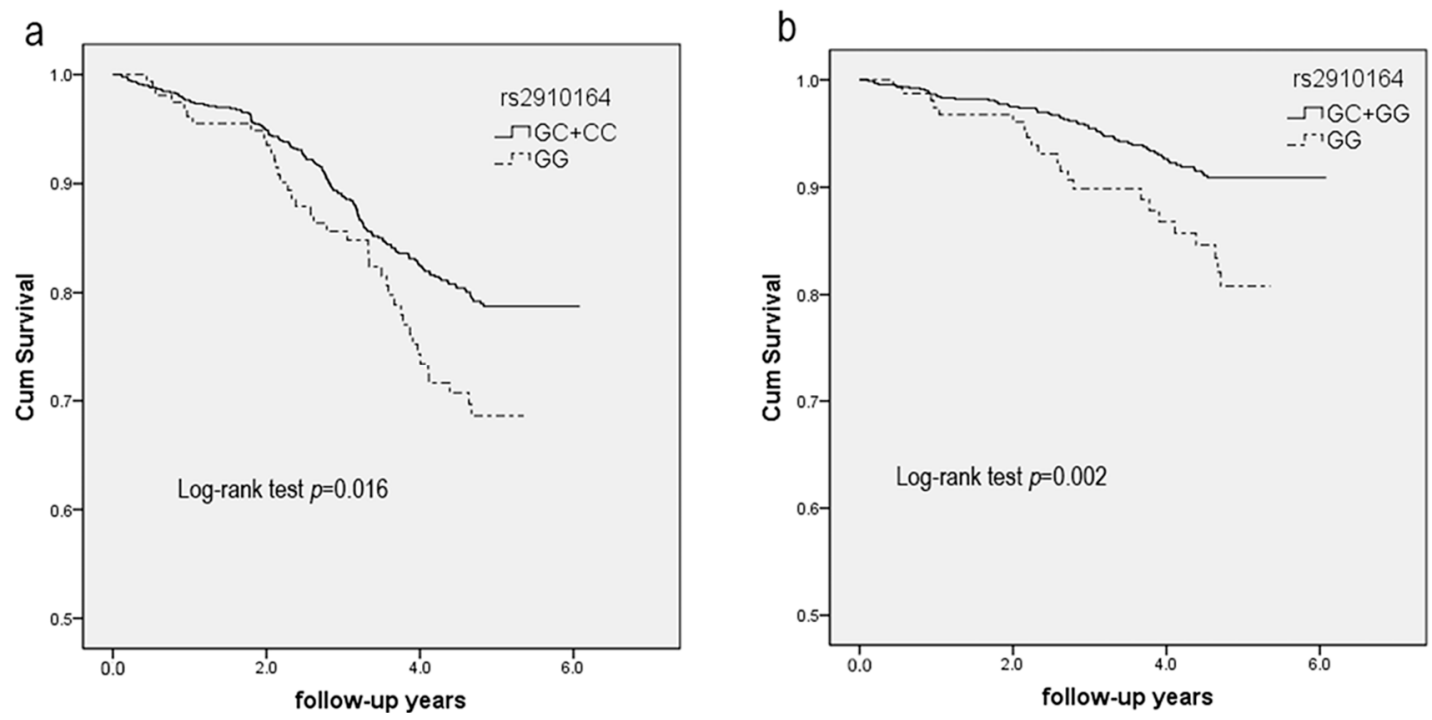

Figure 2. Association between rs2910164 polymorphism and stroke recurrence and Csdeath under the recessive model of inheritance. (a) Effect of the variant rs2910164 on stroke recurrence in patients with ischemic stroke. Kaplan-Meier estimates of the cumulative recurrence-free probability in ischemic stroke patients based on the C allele of rs2910164 polymorphism (log-rank statistic $\chi^{2}=5.796, p=0.016$ ); (b) Effect of the variant rs2910164 on Csdeath in ischemic stroke patients. Kaplan-Meier estimates of the cumulative event-free survival probability in ischemic stroke patients based on the C allele of rs2910164 polymorphism (log-rank statistic $\left.\chi^{2}=9.155, p=0.002\right)$. 
Table 5. rs2910164 GG genotype increased risk of stroke recurrence and Csdeath.

\begin{tabular}{|c|c|c|c|c|c|c|c|c|}
\hline \multirow{2}{*}{ Genotype } & \multirow{2}{*}{$\begin{array}{l}\text { No. of } \\
\text { Patients }\end{array}$} & \multirow{2}{*}{$\begin{array}{l}\text { No. of } \\
\text { Events }\end{array}$} & \multicolumn{2}{|c|}{ Model 1} & \multicolumn{2}{|c|}{ Model 2} & \multicolumn{2}{|c|}{ Model 3} \\
\hline & & & HR (95\% CI) & $p$-Value & HR (95\% CI) & $p$-Value & HR (95\% CI) & $p$-Value \\
\hline \multicolumn{9}{|l|}{ Recurrence } \\
\hline $\mathrm{CC}+\mathrm{GC}$ & 912 & 156 & 1.00 & 0.017 & 1.00 & 0.014 & 1.00 & 0.013 \\
\hline GG & 159 & 40 & $1.53(1.08-2.16)$ & & $1.54(1.09-2.18)$ & & $1.56(1.10-2.20)$ & \\
\hline \multicolumn{9}{|l|}{ Csdeath } \\
\hline $\mathrm{CC}+\mathrm{GC}$ & 912 & 63 & 1.00 & 0.003 & 1.00 & 0.003 & 1.00 & 0.002 \\
\hline GG & 159 & 22 & $2.08(1.28-3.38)$ & & $2.11(1.30-3.42)$ & & $2.13(1.31-3.46)$ & \\
\hline
\end{tabular}

\section{Discussion}

In this study, we investigated the association of the miR-146a rs2910164 SNP with stroke incidence and prognosis. Our large-scale prospective investigation showed that rs2910164 was associated with a 1.56-fold increased risk of stroke recurrence and a 2.13-fold increased risk of Csdeath in a Chinese population. However, we observed no association with ischemic stroke incidence. To the best of our knowledge, it is the first time we found the association between rs2910164 with ischemic stroke prognosis.

There are a number of possible mechanisms to explain why rs2910164 is a significant predictor of stroke prognosis. rs2910164 involves a C-to-G nucleotide substitution, which can cause a C:U pair change to a G:U mismatch in the stem structure of the miR-146a precursor, and therefore decreases the expression of mature miR-146a. miR-146a regulates a pathway and it can accelerate the binding of the transcriptional repressor RelB to the TNF- $\alpha$ promoter [28]. miR-146a primarily targets IRAK1 and TRAF6, resulting in the inhibition of nuclear factor (NF)- $\mathrm{KB}$ via the Toll-like receptor pathway [29]. Therefore, the down-regulation of miR-146a may increase inflammation-related atherosclerosis and affect vascular damage response by increasing the levels of TNF- $\alpha$, TRAF6, and IRAK1. Overexpression of miR-146a in peripheral blood mononuclear cells activates Th1 cells and induces the expression of TNF- $\alpha$, monocyte chemotactic protein $1, N F-\kappa B$, and p65 through post-transcriptional enhancement of the T-bet pathway [30].

Previously, rs2910164 was significantly associated with ischemic stroke prevalence and increased stroke risk in female, normotensive, and nondiabetic groups in a South Korean population [31]. However, we found no association between rs2910164 and ischemic stroke incidence. A previous investigation by Zhu et al. observed that rs2910164 had a protective role against the incidence of large-artery atherosclerotic stroke in the northern Chinese Han population [32], although Liu et al. failed to find any relationship between rs2910164 and ischemic stroke [33]. These different findings could reflect ethnic variations and limitations of sample sizes, but most important, the sex, subtype of stroke may contribute a lot to this. For this reason, we have analyzed the association between rs 2910164 and ischemic stroke incidence based on stroke subtype and sex, and we conducted a meta-analysis of the four above mentioned studies, but the result showed no relationship between rs2910164 and ischemic stroke incidence.

The current study has a number of limitations. Although the sample size was relatively large, a larger patient population involving other ethnicities should be included. Additionally, in our meta-analysis, the absence of original data restricted us to further evaluate gene-gene and gene-environment interactions. Independent genetic studies should also be performed to detect levels of mature miR-146a and to elucidate novel target genes and regulatory molecules. In addition, we performed analyses for several genetic models, namely, dominant, recessive, codominant, and additive ones; the results showed that the dominant model and recessive model are the most appropriate. 


\section{Materials and Methods}

\subsection{Study Population}

Patients in the case-control study were recruited from among participants of the previously described Multicenter Chinese Stroke Study [34]. Between November 2000 and November 2001, we consecutively recruited 1139 ischemic stroke patients from seven clinical centers (Beijing, Tianjin Yanzhou, Xi'an, Wuhan Xiehe, Wuhan Tongji, and Chongqing, China) together with 1585 age- and sex-matched control subjects. To minimize phenotypic heterogeneity, we recruited patients with only one of the two subtypes of ischemic stroke (cerebral thrombosis and lacunar infarct).

We used neurological examination, magnetic resonance imaging (MRI), or computed tomography (CT) to confirm stroke, in strict accordance with the criteria of the International Classification of Diseases (9th revision). It was defined as a sudden onset of nonconvulsive and focal neurological deficits persisting for $>24 \mathrm{~h}$. We excluded patients who had other kinds of stroke: subarachnoid hemorrhage, cerebrovascular malformation, embolic brain infarction, brain tumors, and transient ischemic attack, and other comorbidity diseases such as: inflammation, collagenosis, liver, metabolic disease, tumorous, or renal disease.

Our investigations were conducted consistent with the rules of the Declaration of Helsinki, and identified by the ethics committee and institutional review board of Fuwai Hospital. All participants were given written informed consent.

\subsection{Follow-up and Outcome Assessment in a Prospective Study}

Of the 1139 ischemic stroke patients, 1071 were recruited after an average 4.5 years of follow-up until 2006 by physicians through the administration of a standard questionnaire and telephone contact. Endpoints included stroke recurrence and Csdeath. Recurrent stroke was defined as a new-onset acute new focal neurological deficit without obvious cause other than vascular disease origin after stroke or an acute aggravation of the existing focal neurological deficit without obvious cause other than vascular disease which was occurring at least 21 days after the first stroke [35]. Deaths were reported by family members. No significant differences in the frequency of genotypes and clinical parameters of patients were found between those who were followed up and those who were lost to follow-up. The event-free group comprised those patients who had no events and were unable to be followed up completely.

\subsection{Measurements of Biochemical Parameters and Collection of Clinical Data}

Routine clinical interviews were conducted to ascertain each patient's history of hypertension, diabetes mellitus, cigarette smoking, and alcohol intake. The 12-h overnight fasting blood of these patients were collected. In patients with acute medical events, we delayed the collection of blood samples by six weeks. Plasma was separated by centrifugation and the white blood cell buffy coat was stored at $-80^{\circ} \mathrm{C}$. Biochemical variables, including total plasma cholesterol, triglyceride, high-density-lipoprotein cholesterol levels, and blood glucose, were determined by an automatic Hitachi 7060 chemistry analyzer (Hitachi, Tokyo, Japan).

\subsection{Genotyping Variants of rs2910164}

Ligase detection reaction (LDR) was used to genotype rs2910164 variants (Shanghai Biowing Applied Biotechnology Co., Ltd: Shanghai, China). Primer or probe sequences and PCR or LDR product lengths of the variants are listed in Table 6. Fragment was amplified in $20-\mu \mathrm{L}$ multiplex PCR reaction mixtures including $50 \mathrm{ng}(1 \mu \mathrm{L})$ of genomic DNA, $2 \mu \mathrm{L}$ of dNTPs, $2 \mu \mathrm{L}$ of $1 \times$ buffer, $0.6 \mu \mathrm{L}$ of $\mathrm{Mg}^{2+}, 0.2 \mu \mathrm{L}$ of Taq polymerase, $4 \mu \mathrm{L}$ of $1 \times \mathrm{Q}$-solution, $0.4 \mu \mathrm{L}$ of primer mix, and $9.8 \mu \mathrm{L}$ of $\mathrm{ddH}_{2} \mathrm{O}$, using the thermal cycler Gene Amp PCR system 9600 (Perkin Elmer, Waltham, MA, USA). Further amplification was conducted in 10- $\mu \mathrm{L}$ multiplex LDR reaction mixtures containing $100 \mathrm{ng}$ of 
original PCR product $(1 \mu \mathrm{L}), 0.05 \mu \mathrm{L}$ of NEB Taq DNA ligase, $1 \mu \mathrm{L}$ of probe mix, and $6.95 \mu \mathrm{L}$ of $\mathrm{ddH}_{2} \mathrm{O}$, using the ABI PRISM 377 DNA Sequencer detected and GeneMapper software (ABI) analyzed.

Table 6. Primer or probe sequences and PCR or LDR product length of rs2910164.

\begin{tabular}{cclc}
\hline Variants & Primer or Probe & \multicolumn{1}{c}{ Sequence $\mathbf{( 5}^{\prime} \mathbf{- 3}^{\prime} \mathbf{)}$} & PCR or LDR \\
\hline & rs2910164-up & CTGGACTGCAAGGAGGGGTCTT & 151 \\
rs2910164G/C & rs2910164_mow & GTCCTCAAGCCCACGATGACAG & P-TGAAATTCAGTTCTTCAGCTGGGATTT-FAM \\
& rs2910164_G & TTCCGCGTTCGGACTGATATCATGGGTTGTGTCAGTGTCAGACATG & 94 \\
& rs2910164_C & TACGGTTATTCGGGCTCCTGTCATGGGTTGTGTCAGTGTCAGACATC & 95 \\
\hline
\end{tabular}

Abbreviations: P, phosphorylated; FAM, Carboxyfluorescein.

\subsection{Statistical Analysis}

The $\chi^{2}$ test was performed to test genotype and allele frequencies, qualitative variables, and HWE. Multivariable logistic regression model was conducted to evaluate associations between rs2910164 and stroke incidence. In the prospective study, Kaplan-Meier survival analysis and Cox proportional hazards models were used to describe the association between rs2910164 and stroke recurrence and prognosis. The unadjusted hazard ration was shown in Model 1. In Model 2, age and sex were adjusted, and age, sex, hypertension, diabetes mellitus, smoking status, and alcohol intake were adjusted in Model 3. Two-tailed $p<0.05$ was considered significant. Statistical analyses were carried out with SPSS software, version 13.0 (SPSS Inc., Chicago, IL, USA).

\subsection{Meta-Analysis}

A meta-analysis of four studies on the role of rs2910164 in ischemic stroke, including our case-control study, two other studies in Chinese populations, and one in a South Korean population, was performed (Table 7). A total of 2481 ischemic stroke patients and 2910 controls were analyzed. Literature was searched in PubMed and EMBASE databases, using the following retrieval strategy: miR-146a AND polymorphisms AND stroke incidence. The inclusion criteria are as followed: (1) the association between the miR146a rs2910164 polymorphism and ischemic stroke are case-control studies; (2) containing original data; and (3) following HWE. Exclusion criteria were: (1) study design other than case-control; (2) not reporting genotypic and allelic frequencies; and (3) family members studied based on linkage considerations. Two investigators discussed to resolve the disagreements between them. We calculated odds ratio (OR), 95\% confidence interval $(95 \% \mathrm{CI})$ through the fixed effects model or DerSimonian-Laird and random effects model to describe the relationship between rs2910164 and stroke incidence. Statistical analysis was carried out using Stata (9th edition; Stata Corporation, College Station, TX, USA) and RevMan (5th edition) software.

Table 7. Association between rs2910164 polymorphism and ischemic stroke incidence in Asian populations (four studies).

\begin{tabular}{cccc}
\hline Study & Case & Control & OR, 95\%CI \\
\hline Our study & 1139 & 1585 & $0.97(0.82-1.14)$ \\
Jeon, Y.J.; et al. [31] & 678 & 553 & $1.26(1.00-1.59)$ \\
Zhu, R.; et al. [32] & 368 & 381 & $0.82(0.61-1.10)$ \\
Liu, Y.; et al. [33] & 296 & 391 & $1.05(0.75-1.46)$ \\
Total & 2481 & 2910 & $1.01(0.90-1.14)$ \\
\hline Heterogeneity: $\chi^{2}=5.67, p=0.129, I^{2}=47.1 \%$.
\end{tabular}

\section{Conclusions}

We found that rs2910164 increased the risk of stroke recurrence and Csdeath in a Chinese population but did not predict stroke incidence in Asian populations, suggesting that it has the 
potential to be a target for therapeutic interventions that aim to reduce inflammation and improve stroke outcome.

Acknowledgments: We thank the technicians at the Sino-German Laboratory for Molecular Medicine, Fuwai Hospital, for their technical support, and the staff members of the Multicenter Stroke Study for assisting with the data collection. This study was supported by the 973 program (2014CB541601, Jingzhou Chen) and the National Natural Science Foundation of China (91539113, Jingzhou Chen).

Author Contributions: Jing-Zhou Chen and Jie Xi conceived of and designed the experiments; Yin-Hui Zhang, Chan-Na Zhang, and Li Song performed the experiments; Ru-Tai Hui and Jiao-Yan Qu analyzed the data; Yan Song contributed reagents/materials/analysis tools; and Jiao-Yan Qu and Jie Xi wrote the paper.

Conflicts of Interest: The authors declare no conflicts of interest.

\section{References}

1. Liu, L.; Wang, D.; Wong, K.S.; Wang, Y. Stroke and stroke care in china: Huge burden, significant workload, and a national priority. Stroke 2011, 42, 3651-3654. [CrossRef] [PubMed]

2. Liu, M.; Wu, B.; Wang, W.Z.; Lee, L.M.; Zhang, S.H.; Kong, L.Z. Stroke in china: Epidemiology, prevention, and management strategies. Lancet. Neurol. 2007, 6, 456-464. [CrossRef]

3. Jia, Q.; Liu, L.P.; Wang, Y.J. Stroke in China. Clin. Exp. Parmacol. Physiol. 2010, 37, 259-264. [CrossRef] [PubMed]

4. Ma, Y.; Liu, Y.; Fu, H.M.; Wang, X.M.; Wu, B.H.; Wang, S.X.; Peng, G.G. Evaluation of admission characteristics, hospital length of stay and costs for cerebral infarction in a medium-sized city in china. Eur. J. Neurol. 2010, 17, 1270-1276. [CrossRef] [PubMed]

5. Dominiczak, A.F.; McBride, M.W. Genetics of common polygenic stroke. Nat. Genet. 2003, 35, 116-117. [CrossRef] [PubMed]

6. Goldstein, L.B.; Adams, R.; Becker, K.; Furberg, C.D.; Gorelick, P.B.; Hademenos, G.; Hill, M.; Howard, G.; Howard, V.J.; Jacobs, B.; et al. Primary prevention of ischemic stroke: A statement for healthcare professionals from the stroke council of the American heart association. Stroke 2001, 32, 280-299. [CrossRef] [PubMed]

7. Bersano, A.; Ballabio, E.; Bresolin, N.; Candelise, L. Genetic polymorphisms for the study of multifactorial stroke. Hum. Mutat. 2008, 29, 776-795. [CrossRef] [PubMed]

8. Zhang, Z.; Xu, G.; Zhu, W.; Cao, L.; Bai, W.; Xiong, Y.; Yan, B.; Liu, X. Chromosome 12p13 variants predict recurrence of ischaemic stroke in a Chinese population. Eur. J. Neurol. 2014, 21, 1400-1405. [CrossRef] [PubMed]

9. Bennett, D.; Yan, B. Suboptimal response to clopidogrel: A genetic risk factor for recurrent ischaemic stroke. J. Clin. Neurosci. 2013, 20,767-770. [CrossRef] [PubMed]

10. Bai, Y.; Chen, J.; Sun, K.; Wang, Y.; Hui, R. A functional variant in promoter region of platelet-derived growth factor-D is probably associated with intracerebral hemorrhage. J. Neuroinflamm. 2012, 9, 26. [CrossRef] [PubMed]

11. Bartel, D.P. Micrornas: Genomics, biogenesis, mechanism, and function. Cell 2004, 116, 281-297. [CrossRef]

12. Lagos-Quintana, M.; Rauhut, R.; Lendeckel, W.; Tuschl, T. Identification of novel genes coding for small expressed RNAs. Science 2001, 294, 853-858. [CrossRef] [PubMed]

13. Lu, J.; Getz, G.; Miska, E.A.; Alvarez-Saavedra, E.; Lamb, J.; Peck, D.; Sweet-Cordero, A.; Ebert, B.L.; Mak, R.H.; Ferrando, A.A.; et al. MicroRNA expression profiles classify human cancers. Nature 2005, 435, 834-838. [CrossRef] [PubMed]

14. Zhao, Y.; Srivastava, D. A developmental view of microRNA function. Trends Biochem. Sci. 2007, 32, $189-197$. [CrossRef] [PubMed]

15. Thorsen, S.B.; Obad, S.; Jensen, N.F.; Stenvang, J.; Kauppinen, S. The therapeutic potential of microRNAs in cancer. Cancer J. 2012, 18, 275-284. [CrossRef] [PubMed]

16. Li, C.; Pei, F.; Zhu, X.; Duan, D.D.; Zeng, C. Circulating microRNAs as novel and sensitive biomarkers of acute myocardial infarction. Clin. Biochem. 2012, 45, 727-732. [CrossRef] [PubMed]

17. Jeyaseelan, K.; Lim, K.Y.; Armugam, A. MicroRNA expression in the blood and brain of rats subjected to transient focal ischemia by middle cerebral artery occlusion. Stroke 2008, 39, 959-966. [CrossRef] [PubMed]

18. Tan, K.S.; Armugam, A.; Sepramaniam, S.; Lim, K.Y.; Setyowati, K.D.; Wang, C.W.; Jeyaseelan, K. Expression profile of microRNAs in young stroke patients. PLoS ONE 2009, 4, e7689. [CrossRef] [PubMed] 
19. Rink, C.; Khanna, S. MicroRNA in ischemic stroke etiology and pathology. Physiol. Genom. 2011, 43, 521-528. [CrossRef] [PubMed]

20. Tan, J.R.; Koo, Y.X.; Kaur, P.; Liu, F.; Armugam, A.; Wong, P.T.; Jeyaseelan, K. MicroRNAs in stroke pathogenesis. Curr. Mol. Med. 2011, 11, 76-92. [CrossRef] [PubMed]

21. Wang, D.G.; Fan, J.B.; Siao, C.J.; Berno, A.; Young, P.; Sapolsky, R.; Ghandour, G.; Perkins, N.; Winchester, E.; Spencer, J.; et al. Large-scale identification, mapping, and genotyping of single-nucleotide polymorphisms in the human genome. Science 1998, 280, 1077-1082. [CrossRef] [PubMed]

22. Duan, R.; Pak, C.; Jin, P. Single nucleotide polymorphism associated with mature miR-125a alters the processing of pri-miRNA. Hum. Mol. Genet. 2007, 16, 1124-1131. [CrossRef] [PubMed]

23. Ryan, B.M.; Robles, A.I.; Harris, C.C. Genetic variation in microRNA networks: The implications for cancer research. Nature reviews. Cancer 2010, 10, 389-402. [PubMed]

24. Jeon, Y.J.; Choi, Y.S.; Rah, H.; Kim, S.Y.; Choi, D.H.; Cha, S.H.; Shin, J.E.; Shim, S.H.; Lee, W.S.; Kim, N.K. Association study of microRNA polymorphisms with risk of idiopathic recurrent spontaneous abortion in Korean women. Gene 2012, 494, 168-173. [CrossRef] [PubMed]

25. Min, K.T.; Kim, J.W.; Jeon, Y.J.; Jang, M.J.; Chong, S.Y.; Oh, D.; Kim, N.K. Association of the miR-146ac>g, $149 \mathrm{c}>\mathrm{t}, 196 \mathrm{a} 2 \mathrm{c}>\mathrm{t}$, and 499a $>$ g polymorphisms with colorectal cancer in the Korean population. Mol. Carcinog. 2012, 51 (Suppl. 1), E65-E73. [CrossRef] [PubMed]

26. Park, Y.S.; Jeon, Y.J.; Lee, B.E.; Kim, T.G.; Choi, J.U.; Kim, D.S.; Kim, N.K. Association of the miR-146aC>G, $m i R-196 a 2 \mathrm{C}>\mathrm{T}$, and $m i R-499 \mathrm{~A}>\mathrm{G}$ polymorphisms with moyamoya disease in the Korean population. Neurosci. Lett. 2012, 521, 71-75. [CrossRef] [PubMed]

27. Hung, P.S.; Chang, K.W.; Kao, S.Y.; Chu, T.H.; Liu, C.J.; Lin, S.C. Association between the rs2910164 polymorphism in pre-miR-146a and oral carcinoma progression. Oral Oncol. 2012, 48, 404-408. [CrossRef] [PubMed]

28. El Gazzar, M.; Church, A.; Liu, T.; McCall, C.E. MicroRNA-146a regulates both transcription silencing and translation disruption of TNF- $\alpha$ during TLR4-induced gene reprogramming. J. Leukoc. Biol. 2011, 90, 509-519. [CrossRef] [PubMed]

29. Ramkaran, P.; Khan, S.; Phulukdaree, A.; Moodley, D.; Chuturgoon, A.A. miR-146a polymorphism influences levels of miR-146a, IRAK-1, and TRAF-6 in young patients with coronary artery disease. Cell Biochem. Biophys. 2014, 68, 259-266. [CrossRef] [PubMed]

30. Guo, M.; Mao, X.; Ji, Q.; Lang, M.; Li, S.; Peng, Y.; Zhou, W.; Xiong, B.; Zeng, Q. miR-146a in PBMCs modulates th1 function in patients with acute coronary syndrome. Immunol. Cell Biol. 2010, 88, 555-564. [CrossRef] [PubMed]

31. Jeon, Y.J.; Kim, O.J.; Kim, S.Y.; Oh, S.H.; Oh, D.; Kim, O.J.; Shin, B.S.; Kim, N.K. Association of the miR-146a, miR-149, miR-196a2, and miR-499 polymorphisms with ischemic stroke and silent brain infarction risk. Arterioscler. Thromb. Vasc. Biol. 2013, 33, 420-430. [CrossRef] [PubMed]

32. Zhu, R.; Liu, X.; He, Z.; Li, Q. miR-146a and miR-196a2 polymorphisms in patients with ischemic stroke in the northern Chinese Han population. Neurochem. Res. 2014, 39, 1709-1716. [CrossRef] [PubMed]

33. Liu, Y.; Ma, Y.; Zhang, B.; Wang, S.X.; Wang, X.M.; Yu, J.M. Genetic polymorphisms in pre-microRNAs and risk of ischemic stroke in a Chinese population. J. Mol. Neurosci. 2014, 52, 473-480. [CrossRef] [PubMed]

34. Li, Z.; Sun, L.; Zhang, H.; Liao, Y.; Wang, D.; Zhao, B.; Zhu, Z.; Zhao, J.; Ma, A.; Han, Y.; et al. Elevated plasma homocysteine was associated with hemorrhagic and ischemic stroke, but methylenetetrahydrofolate reductase gene $c 677 t$ polymorphism was a risk factor for thrombotic stroke: A multicenter case-control study in China. Stroke 2003, 34, 2085-2090. [CrossRef] [PubMed]

35. Burn, J.; Dennis, M.; Bamford, J.; Sandercock, P.; Wade, D.; Warlow, C. Long-term risk of recurrent stroke after a first-ever stroke. The Oxfordshire Community Stroke project. Stroke 1994, 25, 333-337. [CrossRef] [PubMed]

(C) 2016 by the authors; licensee MDPI, Basel, Switzerland. This article is an open access article distributed under the terms and conditions of the Creative Commons Attribution (CC-BY) license (http://creativecommons.org/licenses/by/4.0/). 